African Journal of Microbiology Research Vol. 6(49), pp. 7494-7503, 25 December, 2012

Available online at http://www.academicjournals.org/AJMR

DOI: $10.5897 / A J M R 12.1509$

ISSN 1996-0808 @2012 Academic Journals

Full Length Research Paper

\title{
Selection of probiotic strains and development of green tea based probiotics for livestock
}

\author{
Md. Elias Hossain ${ }^{1,2}$, Seok Young Koํㅜ, Sang Suk Lee ${ }^{1}$ and Chul Ju Yang ${ }^{1 *}$ \\ ${ }^{1}$ Department of Animal Science and Technology, Sunchon National University, Suncheon, Jeonnam 540-950, \\ South Korea. \\ ${ }^{2}$ Department of Poultry Science, Bangladesh Agricultural University, Mymensingh-2202, Bangladesh.
}

Accepted 27 November, 2012

\begin{abstract}
Twenty six (26) strains of Lactobacillus acidophilus, Lactobacillus plantarum, Lactobacillus casei, Enterococcus faecium, Bacillus subtilis, Bacillus coagulans, and Saccharomyces cerevisiae from Korean Collection for Type Cultures (KCTC) were evaluated in this experiment for the development of green tea based probiotics production and their inhibition effect against pathogenic bacteria was examined. The strains were first tested for acid and bile tolerance, and the higher tolerance strains were subsequently analyzed for heat tolerance and susceptibility to green tea extracts. Among the tested strains, L. acidophilus KCTC 3111, L. plantarum KCTC 3104, B. subtilis KCTC 3239, B. coagulans KCTC 1015, and S. cerevisiae KCTC 7915 which displayed a high tolerance to acid, bile, and heat, and no susceptibility to green tea extract, were selected to produce fermented green tea probiotics. Associative effects of green tea probiotics and Escherichia coli S93 F5, S99 LT, S170 F41, and S171 F6 revealed that E. coli numbers had gradually decreased compared to the control. Stability results of Lactobacillus spp. and Bacillus spp. in green tea probiotics showed that they were stable at $10^{8} \mathrm{CFU} / \mathrm{g}$ during eight weeks of storage. Therefore, it is expected that green tea probiotics may provide a potential feed additive for livestock.
\end{abstract}

Key words: Green tea, probiotics, tolerance, E. coli, feed additive, livestock.

\section{INTRODUCTION}

Probiotics have been defined as mono or mixed cultures of live microorganisms which when administered into diet of animal or man in adequate amounts, make a health benefit to the host (FAO/WHO, 2001). Due to the appearance of antibiotic-resistant bacteria and antibiotic residue in livestock products, the use of probiotics instead of antibiotics has been strongly recommended (Snyder and Champness, 1997). Probiotics have been paid major attention from researchers in recent years. Several studies have been performed to determine the effects of probiotic microorganisms using different

*Corresponding author. E-mail: yangcj@scnu.kr. Tel: 82617503235. Fax: 82617503239. formulas for the purposes of preventing or treating diseases (Mercenier et al., 2002). In animal nutrition, probiotics are used as feed additives as one of the three different groups; lactic acid bacteria (Taheri et al., 2009), Bacillus spores (Anadon et al., 2006), or yeasts (Shareef and Al-Dabbagh, 2009). These groups differ from one another in their properties, origin, and mode of action. The main activities of probiotics are maintenance and reconstitution of intestinal microflora equilibrium which is achieved by various modes of action. The gut microflora have been shown to be involved in protection against a variety of pathogens including Escherichia coli, Salmonella, Campylobacter, Clostridium, and rotavirus. Hence, probiotics may provide an effective preventative and therapeutic treatment for these infections (Fuller, 1999). 
The expected beneficial characteristics of probiotic strains fall into five basic categories: physiological, immunological, metabolic and genetic traits, and technological properties. Potential probiotic strains must include characteristics such as nontoxicity, nonpathogenicity, ability to modulate immune responses, and production of antimicrobial substances (Lim and Im, 2009). In addition, they should be able to survive and proliferate at the target site, resist gastric acid and bile, adhere to the gastrointestinal tract, and antagonize pathogenic bacteria (Mercenier et al., 2002). The use of probiotic bacteria and their metabolites has shown many beneficial effects in cattle, pigs, and chickens. These advantages include the improvement of general health, feed conversion ratios, and growth rate, as well as resistance to diseases (Fuller, 1992).

Another developed non-antibiotic method in the use involves functional medicinal plants. Green tea (Camellia sinensis) has been used for centuries by Korean, Japanese, and Chinese people as an anti-aging herb and its polyphenolic compounds which are mainly composed of seven types of catechins are reported to have, anticarcinogenic (Mukhtar and Ahmad, 1999), antimicrobial (Hara-Kudo et al., 2005), and antioxidant (Nishida et al., 2006) in animals. In addition to human consumption, low-grade green tea has been used as an ingredient in feed for livestock (Cao et al., 2005; Suzuki et al., 2002) with positive effects on animal performance. Recently, there has been a growing interest among researchers and the feed industry to develop a probiotic feed containing a combination of beneficial microbial strains and medicinal plants. As a result, this study was conducted to select appropriate probiotic strains to use with green tea. Tests, such as acid, bile, and heat tolerance, and susceptibility to green tea extract as well as inhibitory effects on pathogenic bacteria, and stability under storage conditions were also conducted.

\section{MATERIALS AND METHODS}

\section{Microbial strains}

A total of 26 strains, including Lactobacillus acidophilus, $L$. plantarum, L. casei, Enterococcus faecium, Bacillus subtilis, B. coagulans, and Saccharomyces cerevisiae were used to select appropriate probiotic strains. These microbes are part of the Korean Collection for Type Cultures (KCTC) and were obtained from the Korea Research Institute of Bioscience and Biotechnology. The culture media for the experimental strains were de Man, Rogosa, and Sharpe (MRS) medium (Difco, Detroit, MI, USA) for Lactobacillus spp., nutrient broth (NB) medium (Difco) for Bacillus spp., and yeast and mold (YM) medium (Difco) for yeast (Table 1).

\section{Tolerance measurement}

To test the acid tolerance of the strains, simulated gastric juice was made according to a slightly altered method of Kobayashi et al.
(1974). Experimental strains were injected into sterilized broth media $(50 \mathrm{ml})$ and then cultivated at $37^{\circ} \mathrm{C}$ for $48 \mathrm{~h}$. Each strain (1 $\mathrm{ml}$ ) was inoculated into simulated gastric juice consisting of NB medium containing $1000 \mathrm{U}$ pepsin/ml adjusted to $\mathrm{pH} 3$ using $1 \mathrm{~N}$ $\mathrm{HCl}(50 \mathrm{ml})$, and also into sterilized NB medium, $\mathrm{pH} 7(50 \mathrm{ml})$ as a control. Lactobacillus spp. and Bacillus spp. were incubated at $37^{\circ} \mathrm{C}$ and yeast at $30^{\circ} \mathrm{C}$ for $3 \mathrm{~h}$. Serial decimal dilutions in MRS agar medium for Lactobacillus spp., nutrient agar (NA) medium for Bacillus spp., and YM agar medium for yeast were prepared and aliquots $(1 \mathrm{ml})$ from the dilutions were then spotted on MRS, NA, or YM agar plates to determine the number of surviving cells. The experiment was repeated three times. Bile tolerance was measured according to a slightly altered method of Park (1999). For analysis of bile tolerance, $1 \mathrm{ml}$ of inoculation liquid of each strain was injected into sterilized NB medium with $0.3 \%$ hog bile acid or into NB medium without hog bile acid as a control. To count bile acid tolerant cells, the same methods were used as for measuring acid tolerance. Only those experimental strains that were confirmed to have strong acid and bile tolerance in the initial tests were selected for heat tolerance testing. Heat tolerance was tested according to a slightly altered method of You et al. (2005). Lactobacillus spp. and Bacillus spp. were treated with heat at $80^{\circ} \mathrm{C}$ for $15 \mathrm{~min}$, and $70^{\circ} \mathrm{C}$ for $5 \mathrm{~min}$ in the case of yeast. Heat tolerant cells were counted using the same method used for acid tolerance described previously.

\section{Susceptibility of selected strains to green tea extract}

According to the method of Yun et al. (1996), green tea was extracted in a water bath at $80^{\circ} \mathrm{C}$ for 30 min by adding $100 \mathrm{ml}$ of distilled water to $100 \mathrm{mg}$ of green tea powder, after which 10, 20, 30, 40 , and $50 \%$ green tea extract solutions were prepared in Tryptic Soy Agar (TSA) media (Difco). Selected strains that had strong acid, bile, and heat tolerance were inoculated $(1 \mathrm{ml})$ into TSA media containing each of the prepared green tea extract solutions and cultured at $37^{\circ} \mathrm{C}$ for $48 \mathrm{~h}$. The cultures were compared to those grown in TSA media without green tea extract (control) and each strain was defined as 'resistant (+)' if it grew well, 'intermediate (w)' if it did not grow well, or 'susceptible (-)' if it did not grow at all.

\section{Production of green tea probiotics}

Strains selected for tolerance to acid, bile, heat, and green tea extract were used for preparation of green tea probiotics. The process of producing probiotics in this experiment was the same method as applied in industry. We used a commercial fermentor (Model: W-1000, Wonbalhyo Industry Co., Korea) for anaerobic and aerobic fermentation process. Defatted rice bran and wheat bran, which are typically used in the industrial field, were used for fermented solid media (Lee, 2006). Green tea probiotics were produced as follows: L. acidophilus KCTC 3111 and L. plantarum KCTC 3104 were formulated into media containing 30\% green tea, $50 \%$ defatted rice bran, and $20 \%$ wheat bran. The culture was fermented at $40^{\circ} \mathrm{C}$ repeating $5 \mathrm{~h}$ of static and $3 \mathrm{~h}$ of shaking conditions for two days. There was a second inoculation with $B$. subtilis KCTC $3239, B$. coagulans KCTC 1015, and S. cerevisiae KCTC 7915, and continued the process for three days. The number of cells in the green tea probiotics was analyzed as well. The green tea probiotics $(1 \mathrm{~g})$ were diluted with sterilized distilled water $(10 \mathrm{ml})$ at room temperature for approximately $10 \mathrm{~min}$, then $1 \mathrm{ml}$ was diluted 10 -fold serially in $0.85 \% \mathrm{NaCl}$ solution, cultured, and the number of colonies counted. Each strain inoculation was made with $10^{10} \mathrm{CFU} / \mathrm{g}$. 
Table 1. Media and culture methods used for selection of microbial strains.

\begin{tabular}{|c|c|c|c|}
\hline Microbial strain ${ }^{1}$ & Medium & Culture method & Culture time $(\mathrm{h})$ \\
\hline Lactobacillus acidophilus KCTC 3111 & MRS & Anaerobic & 48 \\
\hline \multicolumn{4}{|l|}{ L. acidophilus KCTC 3146} \\
\hline \multicolumn{4}{|l|}{ L. acidophilus KCTC 3149} \\
\hline \multicolumn{4}{|l|}{ L. acidophilus KCTC 3150} \\
\hline Lactobacillus casei KCTC 2180 & MRS & Anaerobic & 48 \\
\hline \multicolumn{4}{|l|}{ L. casei KCTC 3109} \\
\hline Lactobacillus plantarum KCTC 1048 & MRS & Anaerobic & 48 \\
\hline \multicolumn{4}{|l|}{ L. plantarum KCTC 3099} \\
\hline \multicolumn{4}{|l|}{ L. plantarum KCTC 3104} \\
\hline \multicolumn{4}{|l|}{ L. plantarum KCTC 3107} \\
\hline Enterococcus faecium KCTC 2022 & MRS & Anaerobic & 48 \\
\hline \multicolumn{4}{|l|}{ E. faecium KCTC 3078} \\
\hline \multicolumn{4}{|l|}{ E. faecium KCTC 3080} \\
\hline \multicolumn{4}{|l|}{ E. faecium KCTC 3122} \\
\hline Bacillus subtilis KCTC 1022 & NB & Aerobic & 48 \\
\hline \multicolumn{4}{|l|}{ B. subtilis KCTC 1103} \\
\hline \multicolumn{4}{|l|}{ B. subtilis KCTC 1666} \\
\hline \multicolumn{4}{|l|}{ B. subtilis KCTC 3239} \\
\hline Bacillus coagulans KCTC 1013 & NB & Aerobic & 48 \\
\hline \multicolumn{4}{|l|}{ B. coagulans KCTC 1014} \\
\hline \multicolumn{4}{|l|}{ B. coagulans KCTC 1015} \\
\hline \multicolumn{4}{|l|}{ B. coagulans KCTC 1662} \\
\hline Saccharomyces cerevisiae KCTC 1201 & YM & Anaerobic & 24 \\
\hline \multicolumn{4}{|l|}{ S. cerevisiae KCTC 7107} \\
\hline \multicolumn{4}{|l|}{ S. cerevisiae KCTC 7915} \\
\hline S. cerevisiae KCTC 7928 & & & \\
\hline
\end{tabular}

${ }^{1}$ Korean Collection for Type Cultures (KCTC) strains obtained from the Korea Research Institute of Bioscience and Biotechnology (KRIBB). MRS = de Man, Rogosa and Sharpe; NB = nutrient broth; YM = yeast and mold (Difco, USA).

\section{Chemical composition analysis}

The green tea used in this experiment was provided by the Tea Experimental Station (Bosung, Korea). All proximate components of green tea and green tea probiotic were determined by the methods of the Association of Official Analytical Chemists (AOAC, 1990). The method devised by Ikeda et al. (2003) was used for catechin analysis. Briefly, approximately $100 \mathrm{mg}$ of green tea and green tea probiotic samples were dissolved in $100 \mathrm{ml}$ of distilled water and heated in a water bath at $80^{\circ} \mathrm{C}$ for $30 \mathrm{~min}$. After cooling, the solution was filtered and $25 \mathrm{ml}$ was transferred to a separating funnel and mixed three times with $25 \mathrm{ml}$ of chloroform. The water layer was collected and extracted with $25 \mathrm{ml}$ of ethyl acetate. The ethyl acetate extract was then transferred to a rotary evaporator and evaporated at $30^{\circ} \mathrm{C}$ and $5 \mathrm{ml}$ of methanol was added after which it was filtered through a membrane filter (0.45 $\mu \mathrm{m}$ PVDF) and Sep-Pack C18 cartridge. The content of catechin compounds from the extracts was determined using a high pressure liquid chromatography (HPLC, Model 501; Waters, Milford, MA, USA).

\section{Growth inhibition of pathogens}

Growth inhibition of pathogens was measured according to a slightly altered method of Park (1999) and Kim et al. (2002). The experiment used four strains of E. coli; S93 F5, S99 LT, S170 F41, and S171 F6 obtained from National Veterinary Research and Quarantine, Korea. Each pathogenic strain was cultured anaerobically at $37^{\circ} \mathrm{C}$ for $24 \mathrm{~h}$ in $50 \mathrm{ml} \mathrm{TSB}$ media (Difco), then $1 \mathrm{ml}$ of culture, $1 \mathrm{~g}$ of green tea probiotic powder, and the selected strains were inoculated together into a new $50 \mathrm{ml}$ volume of sterilized NB media and associatively incubated in anaerobic conditions at $37^{\circ} \mathrm{C}$ for $24 \mathrm{~h}$. E. coli density was measured every $3 \mathrm{~h}$ and results were compared to a control group of $E$. coli that was cultured alone under the same conditions. MacConkey Sorbitol Agar (Difco, Detroit, MI, USA) was used as E. coli selective media. 


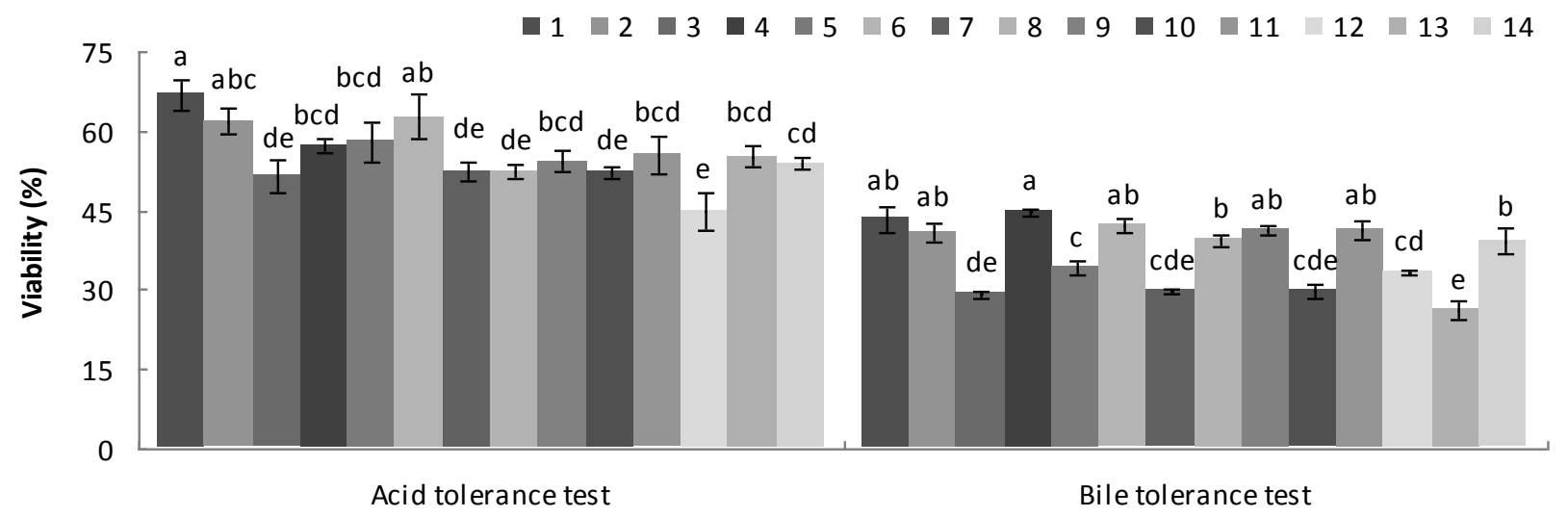

Figure 1. Effect of simulated acid and bile on the growth of lactic acid bacteria. The microbial strains: 1, L. acidophilus KCTC 3111; 2, L. acidophilus KCTC 3146; 3, L. acidophilus KCTC 3149; 4, L. acidophilus KCTC 3150; 5, L. casei KCTC 2180; 6, L. casei KCTC 3109; 7, L. plantarum KCTC 1048; 8, L. plantarum KCTC 3099; 9, L. plantarum KCTC 3104; 10 , L. plantarum KCTC 3107; 1, E. faecium KCTC 2022; 12, E. faecium KCTC 3078; 13, E. faecium KCTC 3080; 14, E. faecium KCTC 3122. Values are expressed as means \pm SE. Bars within the acid or bile tolerance test having uncommon letters indicate significant differences $(a>b>c>d, P<0.05)$.

\section{Stability of probiotic strains under storage conditions}

Manufactured green tea probiotics $(1 \mathrm{~kg})$ were put into vacuum packing and stored for eight weeks at room temperature to estimate microorganism number of green tea probiotics during storage. Every week $1 \mathrm{~g}$ was removed and the number of microorganisms was counted. The same Lactobacillus and Bacillus strains, and defatted rice bran were fermented and compared with green tea probiotics. Serial decimal dilutions in MRS agar medium (Difco) for Lactobacillus spp. and NA medium (Difco) for Bacillus spp. were prepared and aliquots $(1 \mathrm{ml})$ of the dilutions were spotted to determine colony numbers on MRS and NA agar plates, respectively.

\section{Statistical analysis}

All the tests were repeated three times and the data were analyzed using the GLM procedure of SAS (2003). Microbial numbers were logarithmically transformed $\left(\log _{10} \mathrm{CFU} / \mathrm{mL}\right.$ or $\log _{10} \mathrm{CFU} / \mathrm{g}$ ) before analysis. Viability was expressed as percentage and calculated from original microbial numbers, and means were separated by Duncan's multiple range test. All data are presented as mean values $\pm \mathrm{SE}$. The threshold for statistical significance was $\mathrm{P}<0.05$.

\section{RESULTS AND DISCUSSION}

\section{Acid, bile, and heat tolerance of microbial strains}

The effects of simulated gastric juice and bile acids on the growth of lactic acid bacteria are shown in Figure 1. The viability of lactic acid bacteria in simulated gastric juice and bile acid varied among species and strains. $L$. acidophilus KCTC 3111, L. acidophilus KCTC 3146, L. acidophilus KCTC 3150, L. casei KCTC 3109, L. plantarum KCTC 3104, and E. faecium KCTC 2022 exhibited the highest tolerance to acid ( $>50 \%)$ and bile (>40\%). Acid and bile tolerance of Bacillus spp. and S. cerevisiae strains were studied and high survival rates $(>40 \%)$ of $B$. coagulans KCTC 1015 , B. subtilis KCTC 3239 , S. cerevisiae KCTC 7915, and S. cerevisiae KCTC 7928 were observed following exposure to simulated gastric juice and bile acid (Figure 2). A total of 10 strains with relatively strong resistance to acid and bile were selected and tested for heat tolerance (Figure 3). All strains showed their heat tolerance to be between $35.86 \%$ and $54.74 \%$. S. cerevisiae KCTC 7915 exhibited the highest heat tolerance while L. acidophilus KCTC 3146 was lowest.

The microflora of the gastrointestinal tract plays a crucial role in the anatomical, physiological, and immunological development of the host by stimulating the immune system to respond rapidly to infection by pathogens and inhibiting colonization of the gut by harmful or pathogenic bacteria through bacterial antagonism (Cebra et al., 1999). Acid and bile tolerance are two fundamental properties that indicate the ability of probiotic microorganisms to survive passage through the gastrointestinal tract, resisting the acidic conditions in the stomach and the bile acids at the beginning of the small intestine (Strompfová and Lauková, 2007). The results of these tests are predictive of the ability of the strains to survive in acidic products. Garriga et al. (1998) screened lactic acid bacteria with regard to $\mathrm{pH} 3$ tolerance, and showed that $\mathrm{pH} 3$ did not decrease the number of lactic acid bacteria. Tolerance to bile salts was initially associated with the presence of bile salt hydrolase activity (Taranto et al., 2006). The L. plantarum NCIMB 8826 strain was shown to be acid and bile tolerant 


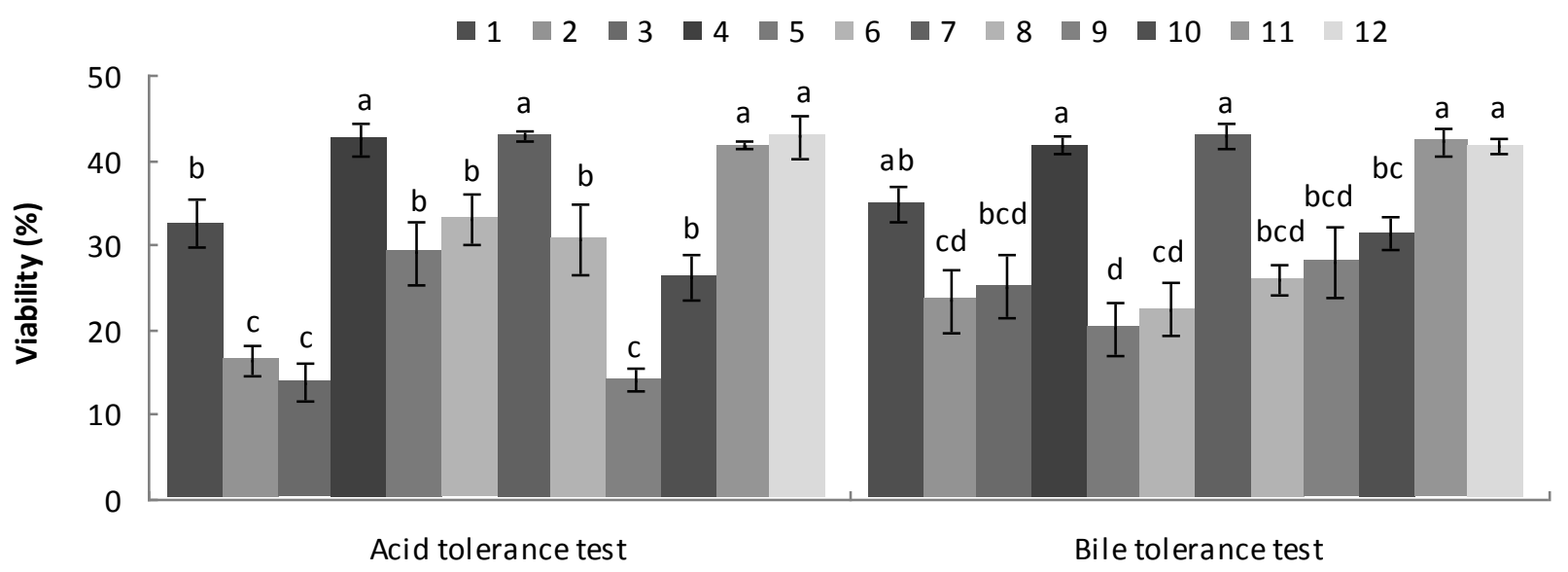

Figure 2. Effect of simulated acid and bile on the growth of Bacillus spp. and Saccharomyces spp. The microbial strains: 1 , B. subtilis KCTC 1022; 2, B. subtilis KCTC 1103; 3, B. subtilis KCTC 1666; 4, B. subtilis KCTC 3239; 5 , B. coagulans KCTC 1013; 6, B. coagulans KCTC 1014; 7, B. coagulans KCTC 1015; 8, B. coagulans KCTC 1662; 9, S. cerevisiae KCTC 1201; 10 , S. cerevisiae KCTC 7107; 11, S. cerevisiae KCTC 7915; 12, S. cerevisiae KCTC 7928. Values are expressed as means \pm SE. Bars within the acid or bile tolerance test having uncommon letters indicate significant differences $(a>b>c>$ d, $P<0.05)$.

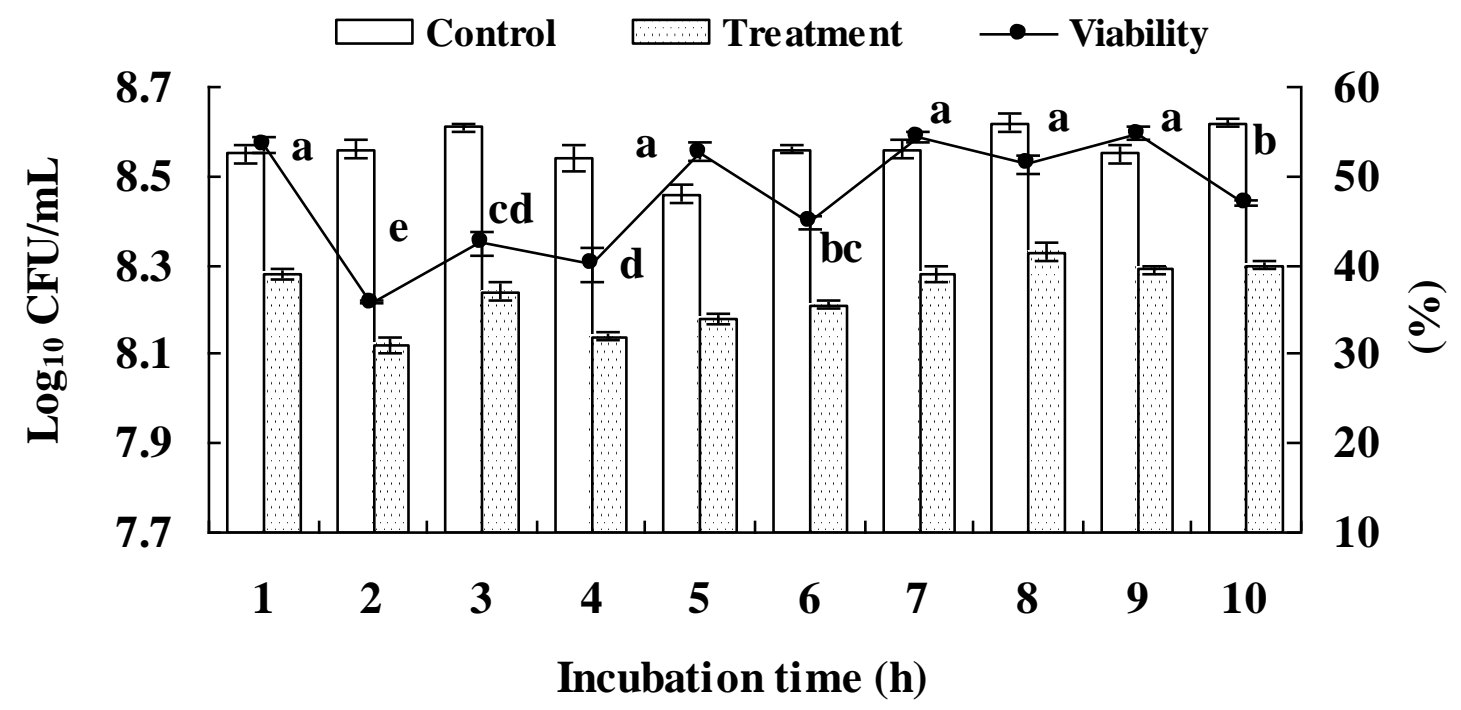

Figure 3. Effect of heat treatment on selected microbial strains. Microbial strain: 1, L. acidophilus KCTC 3111; 2, L. acidophilus KCTC 3146; 3, L. acidophilus KCTC 3150; 4, L. casei KCTC 3109; 5, L. plantarum KCTC 3104; 6, E. faecium KCTC 2022; 7, B. subtilis KCTC 3239; 8, B. coagulans KCTC 1015; 9, S. cerevisiae KCTC 7915; 10, S. cerevisiae KCTC 7928. Values are expressed as means \pm SE. Within the viability of microbial strains, different letters indicate significant differences $(a>b>c>d, P<0.05)$.

(Charalampopoulos and Pandiella, 2010), and has been shown to exert anti-inflammatory activities in animal models (Foligne et al., 2006). Survivability in acid and bile of the strain L. acidophilus (Liong and Shah, 2005), and lactic acid producing bacteria (Thirabunyanon et al., 2009) have been previously reported. Some species of Bacillus are already being used in food production, so their safety has been proven. Some strains of $B$. coagulans are able to survive against heat and acidity of the stomach and bile acids, although in general the strains of this species 
Table 2. Susceptibility of bacterial strains after incubation with green tea extract.

\begin{tabular}{|c|c|c|c|c|c|}
\hline \multirow{2}{*}{ Bacterial strain } & \multicolumn{5}{|c|}{ Green tea extract (\%) } \\
\hline & 10 & 20 & 30 & 40 & 50 \\
\hline L. acidophilus KCTC 3111 & + & + & + & + & + \\
\hline L. acidophilus KCTC 3146 & + & + & + & w & - \\
\hline L. acidophilus KCTC 3150 & + & + & + & w & w \\
\hline L. casei KCTC 3109 & + & + & + & w & - \\
\hline L. plantarum KCTC 3104 & + & + & + & + & + \\
\hline E. faecium KCTC 2022 & + & + & + & w & w \\
\hline B. subtilis KCTC 3239 & + & + & + & + & + \\
\hline B. coagulans KCTC 1015 & + & + & + & + & + \\
\hline S. cerevisiae KCTC 7915 & + & + & + & + & + \\
\hline S. cerevisiae KCTC 7928 & + & + & + & w & w \\
\hline
\end{tabular}

$+=$ resistant; $\mathrm{w}=$ intermediate $;-$ = susceptible .

are quite heterogeneous (De Vecchi and Drago, 2006). Strains with these qualities have an increased chance of survival through the gastrointestinal tract, thus allowing for transient population of the small and large intestines by B. coagulans (Adami and Cavazzoni, 1999). S. cerevisiae is considered as one of the live probiotic microorganisms that are administered through the digestive tract and had a positive impact on the host's health through its direct nutritional effect (Patterson and Burkholder, 2003). Heat treatment is important for the control purposes of pathogens (Leuschner and Bew, 2003). In addition, probiotic strains in feed are required to tolerate heat during the pellet making process (Conway and Kjelleberg, 1989). All selected strains showed average heat tolerance in this study.

\section{Susceptibility of selected microbial strains to green tea extract and chemical composition of green tea probiotics}

Incubation of selected bacterial strains with green tea extract are shown in Table 2. None of the selected strains showed susceptibility to green tea extracts of 10 to $30 \%$, but there was susceptibility of some strains to 40 and $50 \%$ green tea extracts. From this experiment, $L$. acidophilus KCTC 3111 , L. plantarum KCTC 3104 , B. subtilis KCTC 3239, B. coagulans KCTC 1015, and S. cerevisiae KCTC 7915 , which displayed no susceptibility to green tea extracts and had highest heat tolerance $(>50 \%)$, were selected to make fermented green tea probiotics. Each strain inoculation was made with $10^{10} \mathrm{CFU} / \mathrm{g}$ and after fermentation and drying, the number of each strain was reduced 1-2 log cycles (Table 3 ). Chemical analysis results revealed that green tea probiotics had higher levels of moisture and crude ash but lower levels of crude protein, crude fat, crude fiber and catechin content compared to that of green tea alone (Table 3).

None of the selected strains showed susceptibility to green tea extracts of 10 to $30 \%$; however, there was susceptibility to 40 and $50 \%$ extracts. Based on these findings, and consideration of the stability of strains selected to green tea components, we concluded that $30 \%$ would be the proper amount of green tea to use when producing green tea probiotics. From this experiment, $L$. acidophilus KCTC 3111, L. plantarum KCTC 3104, B. subtilis KCTC 3239 , B. coagulans KCTC 1015, and S. cerevisiae KCTC 7915 , which had no susceptibility to green tea extracts, were selected as the strains to make fermented green tea probiotics. It has been shown that some cereals, such as oats and maize, as well as extracts of barley, wheat, and malt are also suitable substrates for the growth of probiotic microorganisms and enhanced acid and bile tolerance (Charalampopoulos and Pandiella, 2010). Although the effects of cereal extracts and cereal fiber on gastrointestinal stability were different, but they displayed a synergistic effect on the gastrointestinal tolerance of Lactobacillus spp. (Michida et al. 2006). Generally, it has been suggested that in animals efficacy for most probiotics could be demonstrated with a daily intake of $10^{8}$ to $10^{9}$ microorganisms (Patterson and Burkholder, 2003). In the study, the number of bacteria in the green tea probiotics was maintained at $10^{8}$ to $10^{9} \mathrm{CFU} / \mathrm{g}$. The total catechin content of green tea probiotics was $30 \%$ of green tea analyzed in this experiment. This result is likely derived from the fact that the mixture ratio of green tea was $30 \%$ in the green tea probiotics.

\section{Growth inhibition of pathogens}

The effects of green tea probiotics on growth inhibition of pathogens after associative inoculation are shown in Figure 4. Following associative inoculation of E. coli S93 F5, S99 LT, S170 F41 and S171 F6 with GTP, the total number of $E$. coli increased up to $6 \mathrm{~h}$, but the numbers were distinctly lower compared to the individual $E$. coli groups. After $6 \mathrm{~h}$ of incubation, E. coli number of the GTP groups dramatically decreased up to the end of the experimental period $(18 \mathrm{~h})$ from 8.41 to $5.85 \log _{10}$ $\mathrm{CFU} / \mathrm{mL}$ for $E$. coli S93 $\mathrm{F} 5$, from 8.50 to $7.12 \log _{10}$ $\mathrm{CFU} / \mathrm{mL}$ for $E$. coli S99 LT, from 8.83 to $5.64 \log _{10}$ $\mathrm{CFU} / \mathrm{mL}$ for $E$. coli S170 F41 and from 8.75 to $6.15 \log _{10}$ $\mathrm{CFU} / \mathrm{mL}$ for $E$. coli S171 F6.

We observed a slow inhibitory effect on $E$. coli proliferation from the beginning when associatively cultured with green tea probiotics, but after $6 \mathrm{~h}$ of inoculation, inhibitory effect was markedly improved. The 
Table 3. The number of microflora and chemical composition of green tea and green tea probiotics.

\begin{tabular}{|c|c|c|}
\hline \multicolumn{2}{|l|}{ Microbial strain } & Microbial number (CFU/g) \\
\hline \multicolumn{2}{|l|}{ Lactobacillus acidophilus KCTC 3111} & $3.2 \times 10^{8} \pm 1.3 \times 10^{7}$ \\
\hline \multicolumn{2}{|l|}{ Lactobacillus plantarum КСТС 3104} & $2.2 \times 10^{8} \pm 1.2 \times 10^{7}$ \\
\hline \multicolumn{2}{|c|}{ Bacillus subtilis KCTC 3239 and Bacillus coagulans КСTC 1015} & $4.5 \times 10^{9} \pm 1.1 \times 10^{8}$ \\
\hline \multicolumn{2}{|c|}{ Saccharomyces cerevisiae KCTC 7915} & $5.2 \times 10^{8} \pm 2.4 \times 10^{7}$ \\
\hline Chemical composition (\%, Dry matter basis) & Green tea & Green tea probiotics \\
\hline Moisture & $11.16 \pm 0.56$ & $15.00 \pm 0.46$ \\
\hline Crude protein & $22.36 \pm 0.55$ & $17.20 \pm 0.45$ \\
\hline Crude fat & $7.36 \pm 0.40$ & $4.93 \pm 0.42$ \\
\hline Crude fiber & $16.20 \pm 0.43$ & $10.89 \pm 0.55$ \\
\hline Crude ash & $6.22 \pm 0.30$ & $7.65 \pm 0.36$ \\
\hline Total catechin & $16.16 \pm 0.43$ & $4.76 \pm 0.33$ \\
\hline
\end{tabular}

Values are expressed as mean \pm SE.

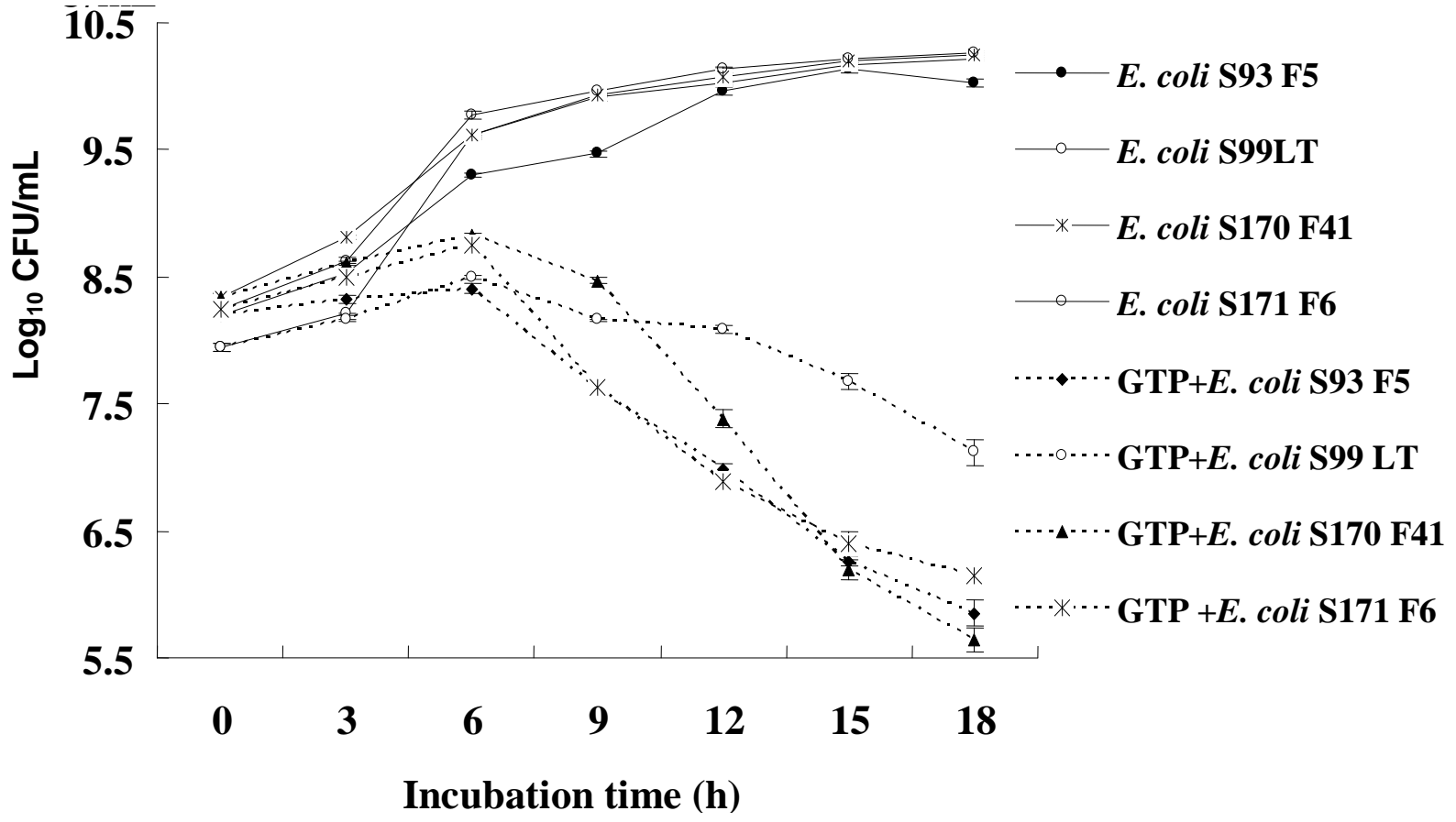

Figure 4. Growth of pathogenic E. coli strains without or with associative inoculation of green tea probiotics. Values are express as means \pm SE. GTP $=$ Green tea probiotics.

rationale behind this observation is that green tea probiotics inhibit pathogens due to the complementary effects of green tea and probiotic bacteria. These results are due to the proliferation of beneficial strains and the release of green tea compounds inside the culture fluid as time passes during the associative culture. It is well known that tea catechins are bactericidal and inhibit the growth of bacterial spores (Hara-kudo et al., 2005), but they do not affect lactic acid bacteria (Hara, 1997). Sugita-Konishi et al. (1999) reported that entero- 


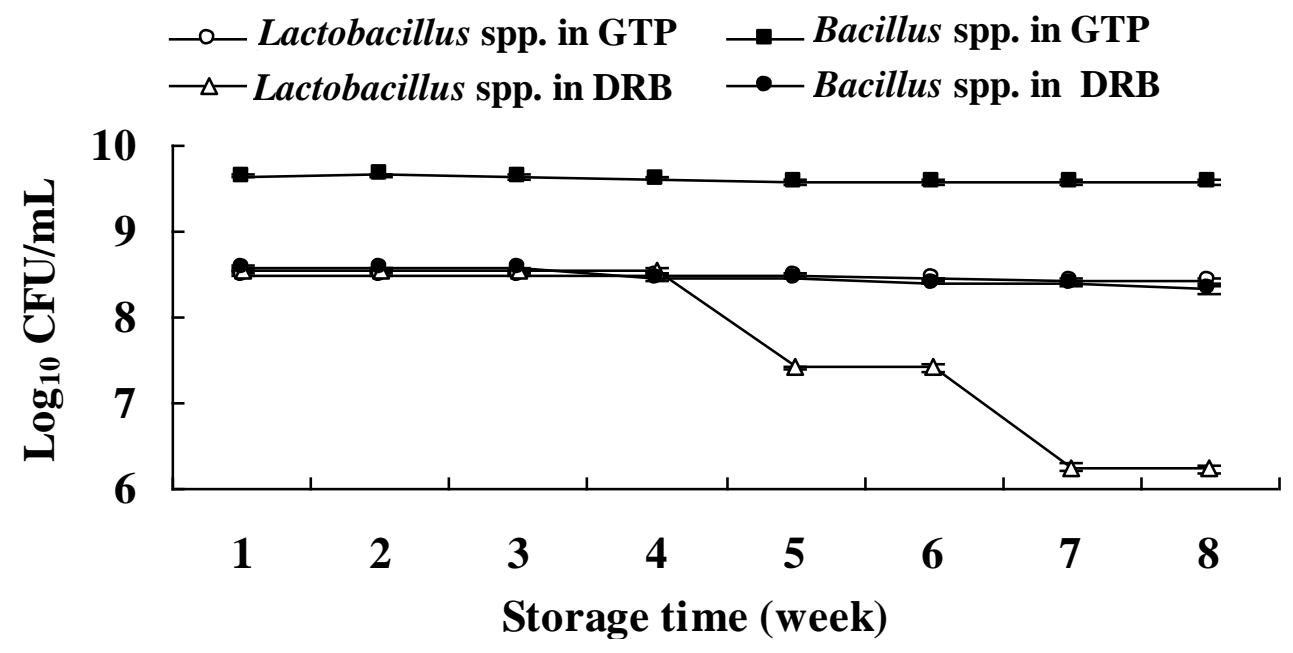

Figure 5. Stability of probiotic strains under storage conditions. Values are express as means \pm SE. GTP = Green tea probiotics; DRB = Defatted rice bran.

hemorrhagic E. coli O157:H7 failed to produce verocytotoxin with low concentrations of green tea catechin. L. acidophilus decreases the $\mathrm{pH}$ of culture solutions and produces the antimicrobial compounds or antibiotics like acidolin, acidophilin, and lactacin-B (Havenaar et al., 1992). Green tea extracts also improved the microflora balance and showed antimicrobial effects against pathogenic bacteria in Holstein calves (Ishihara et al., 2001), and swine (Hara et al., 1995). According to the report of Kim et al. (2002), when L. acidophilus and $E$. coli were inoculated associatively, there was no change for $9 \mathrm{~h}$, but $12 \mathrm{~h}$ after the inoculation, $E$. coli tended to decrease rapidly. Lee et al. (2002) showed that when lactic acid bacterial strains (K1, D2, F35-2) were cocultured with L. monocytogenes, S. typhimurium, or $E$. coli O157:H7 in YS medium, the foodborne microorganisms were completely inhibited within $72 \mathrm{~h}$ of incubation at a ratio of $5: 1$ (isolate:pathogen). Timmerman et al. (2004) noted that multi-strain or multispecies probiotics were more effective than mono-strain probiotics concerning resistance to pathogens and performance in animals.

\section{Stability of probiotic strains under storage conditions}

Changes in the number of microbes in green tea probiotics based on storage period are shown in Figure 5. Lactobacillus spp. with green tea probiotics were stable at $10^{8} \log _{10}$ CFU/g for eight weeks starting from the first week. However, after culture with defatted rice bran, the Lactobacillus spp. were stable for only four weeks, then continuously decreased through eight weeks dropping by approximately 2 log cycles. Bacillus spp. included in defatted rice bran and green tea probiotics maintained a constant number of microbes from the first to the eight weeks of storage.

The survival of probiotics is influenced by various factors, but the storage period strongly affects the survival rate of microbes and the shelf life of products (Hamilton-Miller et al., 1996). The stability of microbes in green tea probiotics may be due to the effects of the green tea fermented medium. In the case of cereal-based fermented products containing probiotic strains, the most likely factors influencing probiotic survival during refrigerated storage are $\mathrm{pH}$ and lactic acid concentration of the fermented product. It has been shown that the levels of sugar and lactic acid in fermented products affect cell survival largely (Charalampopoulos and Pandiella, 2010).

\section{Conclusion}

The green tea probiotics tested in this study were multistrain and exhibited a high tolerance to acid, bile, and heat. Therefore, it is essential to know that selected strains do not inhibit each other in the animal trial. To analyze these results more precisely, more research are needed. In this study, selected strains had no susceptibility to green tea extract and showed stability during eight weeks of storage. In addition, when antimicrobial effect was tested, green tea probiotics inhibited the proliferation of $E$. coli S93 F5, S99 LT, S170 F41, and S171 F6 strains. It is expected from the results that using green tea probiotics as a feed supplement may offer a substitute for the use of antibiotics in livestock nutrition. 


\section{ACKNOWLEDGEMENTS}

The authors would like to thank the Agricultural $R$ \& $D$ Promotion Center (ARPC) of the Korea Rural Economic Institute for their financial assistance.

\section{REFERENCES}

Adami A, Cavazzoni V (1999). Occurrence of selected bacterial groups in the faeces of piglets fed with Bacillus coagulans as probiotic. J. Basic Microbiol. 1:3-9.

Anadon A, Rosa Martinez-Larranaga MR, Martinez MA (2006). Probiotics for animal nutrition in the European Union. Regulation and safety assessment. Regul. Toxicol. Pharmacol. 45:91-95.

AOAC (1990). Official Methods of Analysis 15th edn. Association of Official Analytical Chemist, Arlington, VA, USA.

Cao BH, Karasawa Y, Guo YM (2005). Effects of green tea polyphenols and fructooligosaccharides in semi-purified diets on broiler's performance and caecal microflora and their metabolites. AsianAust. J. Anim. Sci. 18:85-89.

Cebra JJ, Jiang HQ, Šterzl J, Tlaskalova-Hogenova H (1999). The role of mucosal microbiota in the development and maintenance of the mucosal immune system. In: Mucosal Immunology, Academic Press, New York, pp. 267-280.

Charalampopoulos D, Pandiella SS (2010). Survival of human derived Lactobacillus plantarum in fermented cereal extracts during refrigerated storage. LWT-Food Sci. Technol. 43:431-435.

Conway PL, Kjelleberg S (1989). Protein mediated adhesion of Lactobacillus fermentum strain 737 to mouse stomach squamous epithelium. J. Gen. Microbiol. 135:1175-1186.

De Vecchi E, Drago L (2006). Lactobacillus sporogenes or Bacillus coagulans: misidentification or mislabelling? Int. J. Probiotics Prebiotics 1:3-10.

FAO/WHO (2001). Evaluation of health and nutritional properties of probiotics in food including powder milk with live lactic acid bacteria. 2001 Report of a Joint FAO/WHO Expert Consultation. Available from url: $\quad$ www.who.int/foodsafety/ publications/fs management/en/probiotics.pdf.

Foligne B, Nutten S, Steidler L, Dennin V, Goudercourt D, Mercenier A, Pot B (2006). Recommendations for improved use of the murine TNBS-induced colitis model in evaluating anti-inflammatory properties of lactic acid bacteria: technical and microbiological aspects. Digest. Dis. Sci. 51:394-404.

Fuller R (1992). History and development of probiotics. In: Fuller R (eds), Probiotics-The Scientific Basis, Chapman and Hall, London. pp. $1-8$.

Fuller R (1999). Probiotics for farm animals. In: Tannock, GW (eds), Probiotics-A Critical Review, Horizon Scientific, England. pp. 15-22.

Garriga M, Pascual M, Monfort JM, Hugas M (1998). Selection of lactobacilli for chicken probiotic adjuncts. J. Appl. Microbiol. 84:125132.

Hamilton-Miller JM, Shah S, Smith CT (1996). "Probiotic" remedies are not what they seem. Br. Med. J. 312:55-56.

Hara H, Orita N, Hatano S, Ichikawa H, Hara Y, Matsumoto N, Kimura Y, Terada A, Mitsuoka T (1995). Effect of tea polyphenols on fecal flora and fecal metabolic products of pigs. J. Vet. Med. Sci. 57:45-49.

Hara Y (1997). Influence of tea catechins on the digestive tract. J. Cell. Biochem. 27(Suppl.):52-58.

Hara-Kudo Y, Yamasaki A, Sasaki M, Okubo T, Minai Y, Haga M, Kondo K, Sugita-Konishi Y (2005). Antibacterial action on pathogenic bacterial spore by green tea catechins. J. Sci. Food Agric. 85:23542361

Havenaar R, Brink BT, Veld JH (1992). Selection of strains for probiotic use. In: Fuller, R. (eds), Probiotics-The Scientific Basis, Chapman and Hall, New York. pp. 209-223.

Ikeda I, Kobashi M, Hamada T, Tsuda K, Goto H, Imaizumi K, Nozawa A,
Sugimoto A, Kakuda T (2003). Heat-epimerized tea catechins rich in gallocatechin gallate and catechin gallate are more effective to inhibit cholesterol absorption than tea catechins rich in epigallocatechin gallate. J. Agric. Food Chem. 57:7303-7307.

Ishihara N, Chu DC, Akachi S, Juneja LR (2001). Improvement of intestinal microflora balance and prevention of digestive and respiratory organ diseases in calves by green tea extracts. Livest. Prod. Sci. 68:217-229.

Kim EA, Baick SC, Chung WH (2002). A study on growth inhibition of Escherichia coli and Salmonella typhimurium by lactic acid bacteria. J. Anim. Sci. Technol. 44:491-498.

Kobayashi Y, Tohyama K, Terashima T (1974). Studies on biological characteristics of Lactobacillus. II. Tolerance of the multiple antibiotic resistant strain $L$. casei PSR3002 to artificial digestive fluids. Jpn. J. Microbiol. 29:691-697.

Lee JY (2006). Screening of probiotics lactic acid bacteria inhibiting enteropathogenic bacteria, PhD. Thesis. University of Suwon. South Korea.

Lee JY, Park YS, Lee NY, Shin DH (2002). Growth inhibition of some food-borne microorganisms by lactic acid bacteria isolated from feces of newborn baby and from dongchimi. Food Sci. Biotechnol. 11:448456.

Leuschner RGK, Bew J (2003). Enumeration of probiotic bacilli spores in animal feed: Interlaboratory study. J. AOAC Int. 86:568-575.

Lim SM, Im DS (2009). Screening and characterization of probiotic lactic acid bacteria isolated from Korean fermented foods. J. Microbiol. Biotechnol. 19:178-186.

Liong MT, Shah NP (2005). Acid and bile tolerance and cholesterol removal ability of lactobacilli strains. J. Dairy Sci. 88:55-66.

Mercenier A, Pavan S, Pot B (2002). Probiotics as biotherapeutic agents: present knowledge and future prospects. Curr. Pharm. Des. 8:99-110.

Michida H, Tamalampudi S, Pandiella SS, Webb C, Fukuda H, Kondo A (2006). Effect of cereal extracts and cereal fiber on viability of Lactobacillus plantarum under gastrointestinal tract conditions. Biochem. Eng. J. 28:73-78.

Mukhtar H, Ahmad N (1999). Mechanism of cancer chemopreventive activity of green tea. Proc. Soc. Exp. Biol. Med. 220:234-238.

Nishida T, Eruden B, Hosoda K, Nakagawa K, Miyazawa T, Shioya S (2006). Effects of green tea (Camellia sinensis) waste silage and polyethyleneon ruminal fermentation and blood components in cattle. Asian-Aust. J. Anim. Sci. 19:1728-1736.

Park HS (1999). Development of multi-probiotics. The final report of MAF (Ministry of Agriculture and Forestry) research and development project. Korea.

Patterson JA, Burkholder KM (2003). Application of prebiotics and probiotics in poultry production. Poult. Sci. 82:627-631.

SAS (2003). SAS User's Guide, Version 9.1. SAS Institute, Cary, NC, USA.

Shareef AM, Al-Dabbagh ASA (2009). Effect of probiotic (Saccharomyces cerevisiae) on performance of broiler chicks. Iraqi J. Vet. Sci. 2(Suppl. I):23-29.

Snyder L, Champness W (1997). Molecular Genetics of Bacteria 1st edn. American Society for Microbiology, Washington, DC.

Strompfová V, Lauková A (2007). In vitro study on bacteriocin production of Enterococci associated with chickens. Anaerobe 13:228-237.

Sugita-Konishi Y, Hara-Kudo Y, Amano F, Okubo T, Aoi T, Iwaki M, Kumagai S (1999). Epigallocatechin gallate and gallocatechin gallate in green tea catechins inhibit extracellular release of Vero toxin from enterohemorrhagic Escherichia coli O157:H7. Biochim. Biophys. Acta. 1472:42-50.

Suzuki K, Kadowaki H, Hino M, Tamura K (2002). The influence of green tea in pig feed on meat production and quality. Jpn. J. Swine Sci. 39:59-65.

Taheri H, Tabandeh F, Moravej H, Zaghari M, Shivazad M, Shariati P (2009). Potential probiotic of Lactobacillus johnsonii LT171 for chicken nutrition. Afr. J. Biotechnol. 8:5833-5837.

Taranto MP, Perez-Martinez G, Font de Valdez GM (2006). Effect of 
bile acid on the cell membrane functionality of lactic acid bacteria for oral administration. Res. Microbiol. 157:720-725.

Thirabunyanon M, Boonprasom P, Niamsup P (2009). Probiotic potential of lactic acid bacteria isolated from fermented dairy milks on antiproliferation of colon cancer cells. Biotechnol. Lett. 31:571-576.

Timmerman HM, Koningb CJM, Mulderc L, Romboutsd FM, Beynen AC

(2004). Monostrain, multistrain and multispecies probiotics-A comparison of functionality and efficacy. Int. J. Food Microbiol. 96:219-233.
You SJ, Cho JK, Hwang SG, Heo KC (2005). Probiotic characteristics of Lactobacillus rhamnosus isolated from kefir. Korean J. Food Sci. Anim. Resour. 25:357-364.

Yun YP, Kang WS, Lee MY (1996). The antithrombotic effects of green tea catechin. J. Food Hyg. Saf. 11:77-82. 